\title{
Science and society test IX: Technical means of verification
}

\author{
David Hafemeister \\ Program in Science and Technology for International Security, Massachusetts Institute of Technology, \\ Cambridge, Massachusetts, 02139 and Physics Department, California Polytechnic University, San Luis \\ Obispo, California 93407
}

\begin{abstract}
Technical means of monitoring are used to verify compliance to arms control treaties. Numerical estimates are developed in order to quantify some aspects of (1) nuclear testing (signatures, optical double pulse calibration, decoupling in cavities, and high-frequency components in seismic signals), (2) optical reconnaissance (films versus CCDs, air turbulence, adaptive optics, and digital image processing), (3) infrared (resolution and detection sensitivity), and (4) radar (synthetic aperture and missile coefficient).
\end{abstract}

\section{INTRODUCTION}

Over the past several decades there has been remarkable progress in some military technologies such as the increased accuracy of missiles with guidance using stellar locations, Navstar Global Positioning Satellites (GPS), and terrain mapping (TERCOM). At the same time, there has been considerable progress in the technologies which enhance the monitoring of military activities and systems of "the other side." These improvements have been both in quality, with better resolution and data management, and in scope, with new methods of measurement. The technical means of monitoring military endeavors can be extremely important for arms control for three fundamental reasons: (1) They can remove worst case analyses such as that which caused the missile "gap" of 1960, (2) they can allow the US Congress and the Executive branch to have confdence to accept possible arms control treaties, and (3) they can deter leaders from becoming too "adventuresome."

The purpose of this paper is to consider the technical aspects ${ }^{1}$ of some of the means of verification; therefore, it will not include discussions of such topics as the historical, political, and legal aspects of verification; the criteria of the quality of verification ("adequate versus effective"); the possible vulnerability to "breakouts" from arms control treaties; the international means of verification by the International Atomic Energy Agency or the proposed International Satellite Monitoring Agency; various cooperative means of verification where each side helps the other side with its ability to monitor; the specific terms of arms control treaties; and so forth. As in the other papers ${ }^{2}$ in this series of science and society tests, the calculations on the physics of verification will use only widely accepted numerical parameters, and the results are consistent with those from more sophisticated calculations, or with experimental data. In particular, this paper will quantify the following aspects ${ }^{3}$ of the technical means of verification:

(i) Nuclear testing: Detection capabilities implied by the flux of $x$ rays, gamma rays, and neutrons from explosions in space; calibration of the yield of an explosion in the atmosphere from the timing of the optical double pulse; the size of a cavity to decouple an underground nuclear explosion; and the measurement of the high-frequency components of seismic signals to detect decoupling in a cavity.

(ii) Optical reconnaissance: Comparison of the performance of film and charge-coupled devices; defocusing by looking downwards through atmospheric turbulence; resolution with adaptive optics for observing satellites in orbit from the earth; enhancement of images with digital image processing techniques such as the Fourier tansform convolution and addition theroems.

(iii) Infrared monitoring: Resolution and detection sensitivity.

(iv) Radar monitoring: Synthetic aperture radar (SAR) and measurement of the missile coefficient.

\section{NUCLEAR TEST BAN TREATIES}

A Comprehensive Test Ban Treaty (CTBT) which would ban all nuclear tests might slow the development of new weapons systems, would improve the political climate between the superpowers, and would make our weapons policy more consistent with the Nuclear Nonproliferation Treaty (NPT) of 1970 in which the nonnuclear-weapons states have given up their rights to possess and test nuclear weapons. The debate on nuclear test ban treaties has centered on the verifiability of such agreements, as well as the need to test to develop new warhead designs and to maintain the reliability of the weapons stockpile. In this section we would like to address the detection and identification of nuclear weapons tests in space (Sec. II A), in the atmosphere (Sec. II B), and underground (Sec. II C).

\section{A. Tests in space}

The Limited Test Ban Treaty (LTBT) of 1963 forbids the testing of nuclear weapons in space, in the atmosphere, and underwater. In order to monitor these treaties, it is necessary to determine the necesary sensitivity to detect nuclear weapons tests. What are the approximate magnitudes of the neutron, $x$-ray, and prompt gamma-ray fluxes from a $1-\mathrm{kt}$ weapon exploded in space at a distance of $20000 \mathrm{~km}$ from the new Global Positioning Satellites (GPS)? Assume $e^{4}$ that about $70 \%$ of the energy appears as $\mathrm{x}$ rays and $0.3 \%$ as prompt gamma rays, and that the average temperature of the initial fireball in space is about the same as the core of the Sun, $20 \times 10^{6} \mathrm{~K} .\left(1 \mathrm{kt}=10^{12}\right.$ $\mathrm{cal}=4.2 \times 10^{12} \mathrm{~J}$.)

Answer. The yield of a $1-\mathrm{kt}$ weapon is $4.2 \times 10^{12} \mathrm{~J}$, or $2.6 \times 10^{25} \mathrm{MeV}$. If one assumes that each fission event releases about $200 \mathrm{MeV}$ along with one extra neutron that escapes from the weapon, the total number of neutrons that escape from a $1-\mathrm{kt}$ warhead is

$$
\begin{aligned}
N(n) & =\left(2.6 \times 10^{25} \mathrm{MeV}\right)(1 n / 200 \mathrm{MeV}) \\
& =1.3 \times 10^{23} \text { neutrons } .
\end{aligned}
$$


The neutron flux from a weapon of $Y \mathrm{kt}$ at a distance of $R$ $\mathrm{km}$ is

$$
\begin{aligned}
& \left(Y \times 1.3 \times 10^{23}\right) /(4 \pi)\left(R \times 10^{5} \mathrm{~cm}\right)^{2} \\
& =10^{12} Y / R^{2} \text { neutrons } / \mathrm{cm}^{2} .
\end{aligned}
$$

The flux at a distance of $20000 \mathrm{~km}$ from the GPS satellite is $10^{12} / 4 \times 10^{8}=2500$ neutrons $/ \mathrm{cm}^{2} / \mathrm{kt}$.

The flux of energy from the $x$ rays at a distance of $R \mathrm{~km}$ is

$$
\begin{aligned}
U & =(0.7)\left(Y \times 2.6 \times 10^{25} \mathrm{MeV} /(4 \pi)\left(R \times 10^{5} \mathrm{~cm}\right)^{2}\right. \\
& =1.4 \times 10^{14} \mathrm{Y} / R^{2}\left(\mathrm{MeV} / \mathrm{cm}^{2}\right) .
\end{aligned}
$$

If the initial fireball temperature is $20 \times 10^{6} \mathrm{~K}$, the average $\mathrm{x}$-ray energy is about $(\mathrm{kT}=1 / 40 \mathrm{eV}$ at $300 \mathrm{~K})$

$E_{\text {av }} \simeq 3 \mathrm{kT}$

$$
=(3 / 40 \mathrm{eV})\left(20 \times 10^{6} \mathrm{~K} / 300 \mathrm{~K}\right)=5 \mathrm{keV} .
$$

The flux of $\mathrm{x}$ rays at a GPS detector from a 1-kt explosion is

$$
\begin{aligned}
U / E_{\mathrm{av}} & =\left(1.4 \times 10^{14}\right) /\left(2 \times 10^{4} \mathrm{~km}\right)^{2}(0.005 \mathrm{MeV}) \\
& =10^{8} / \mathrm{cm}^{2} .
\end{aligned}
$$

Since $0.3 \%$ of the yield appears in the form of prompt gamma rays and about $70 \%$ of the yield appears as $\mathrm{x}$ rays, the prompt gamma-ray flux is

$$
\begin{aligned}
U & =(0.003 / 0.7)\left(1.4 \times 10^{14}\right) Y / R^{2} \\
& =6 \times 10^{11} Y / R^{2} \mathrm{MeV} / \mathrm{cm}^{2} .
\end{aligned}
$$

These results are in agreement with the results from the Los Alamos Vela program. ${ }^{5}$

\section{B. Tests in the atmosphere}

A nuclear explosion in the atmosphere will give a unique double-peaked optical signature (Fig. 1). The initial burst of $\mathrm{x}$ rays lasts less than $1 \mu \mathrm{s}$; these $\mathrm{x}$ rays are absorbed in the first few meters of air and their energy then "diffuses" to create a fireball by heating ${ }^{4,6}$ the air to about $10^{6} \mathrm{~K}$. Shortly afterwards a shock wave carries the energy beyond the fireball. Since this high-temperature shocked gas is ionized, it is opaque to the optical radiation from the fireball behind it, thus reducing the luminosity of the fireball. After the shocked gas has expanded and cooled, it again becomes transparent to light, and the intensity of the fireball again rises. One can use empirical algorithms from the relative

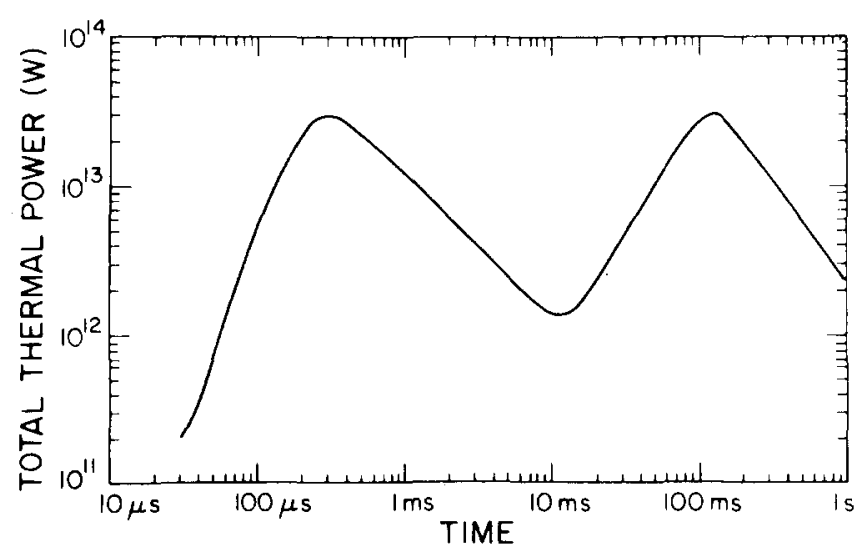

Fig. 1. Optical double pulse from a bhangmeter. The optical double pulse of a 19-kt nuclear weapon exploded in the atmosphere. Figure courtesy of Los Alamos National Laboratory. delay times of the first minimum and second maximum in the light intensity to obtain estimates for the yield of a weapon. Optical "bhangmeters" have been devised to detect double pulses above the background of the intense brightness of the earthshine. Since the hydrodynamical and radiation transport mechanisms of nuclear explosions are very complex, one can only roughly estimate these relative delay times. Estimate the time from the explosion to the minimum light intensity when the shocked region has expanded beyond the fireball ("breakaway") and blocks the radiation from the fireball. The radius of the fireball at "breakaway" is ${ }^{4} R=520 Y^{0.4}$, where $R$ is in meters and $Y$ is in Mt. For the case of $Y=20 \mathrm{kt}$, use an average temperature ${ }^{4,6}$ of $100000 \mathrm{~K}$ during the time regime (ms) of breakaway. The shock front velocity is not very different from the velocity of sound at very high pressures and temperatures.

Answer. The average shock velocity for a 20-kt explosion before "breakaway" is approximately

$$
\begin{aligned}
V(\text { shock }) & \cong V(\text { sound }), \\
& =(331 \mathrm{~m} / \mathrm{s})(100000 \mathrm{~K} / 273 \mathrm{~K})^{1 / 2} \\
& =6400 \mathrm{~m} / \mathrm{s} .
\end{aligned}
$$

The approximate time to the minimum emission at breakway is

$$
t \simeq 520 Y^{0.4} \mathrm{~m} /(6400 \mathrm{~m} / \mathrm{s})=81 Y^{0.4} \mathrm{~ms},
$$

which is in good agreement with the empirical relation given by Brode, ${ }^{6} t=60 Y^{0.4}$ ms. For a 19 -kt weapon, $t \simeq$ (110 $\mathrm{m}) /(6400 \mathrm{~m} / \mathrm{s})=17 \mathrm{~ms}$, which is in good agreement with the data ${ }^{5}$ of Fig. 1. Additional confirmation of the explosion is obtained from coincidence measurements between the activation of the bhangmeter and the arrival of the electromagnetic pulse (EMP) which accompanies a nuclear explosion. Additional evidence will be available from the new GPS system which will observe any spot on the Earth with 4-8 (of the 18) GPS satellites at all times. The accurate clock system of the GPS satellites will allow triangulation methods to determine the position of a nuclear explosion with great accuracy. Had these instruments been functioning in 1979, they would, most likely, have removed the ambiguity from the Vela data of the "event" over the South Atlantic. (In 1980, the Presidential Office of Science and Technology Policy reported that the light signals were probably not from a nuclear explosion.)

\section{Underground tests}

Two of the main arguments that have been raised about the ability to monitor a Complete Test Ban (CTB) are as follows: (1) The nation that desired to cheat might dig a large cavity for its nuclear test so that the nuclear explosion would be relatively decoupled from the earth, thus reducing the amplitude of the seismic signal by as much as a factor of 60 to 200. (2) Because earthquakes and nuclear explosions create fairly similar seismic signals, it is necessary to distinguish nuclear explosions from earthquakes. By using seismic magnitudes based both on short-period body waves $\left(m_{b}\right)$ and on long-period surface waves $\left(\boldsymbol{M}_{s}\right)$, several seismologists have distinguished nuclear tests from earthquakes as well as estimated yields of underground explosions. In order to maximize the capability to verify a CTB, each side must be able to observe seismic signals on the soil of the other country. Starting in the mid-1960s, The 
Sandia National Laboratory built and tested unattended seismic stations, ${ }^{7}$ and now uses them in a network in the US, Canada, and Norway. A network of about 40 stations located inside and adjacent to the USSR could distinguish earthquakes from nuclear tests with yields as low as about ${ }^{8}$ $1 \mathrm{kt}$. The close proximity of the in-country stations would improve the capability to observe the higher frequencies (up to $30 \mathrm{~Hz}$ or higher). Identification of the type of source of the signal is based ${ }^{8,9}$ on the fact that explosions have a greater fraction of their energy content at higher frequencies than do earthquakes.

\section{Cavity size}

Consider a nuclear explosion of yield $Y$ in a cavity in salt such that the pressure from the explosion at a distance $(a)$ does not exceed the elastic limit $\left(P_{0}\right)$ of the medium. How large a cavity would be needed to decouple a 1-kt explosion? Assume the elastic limit for salt is $P_{0}=\mathbf{4 4 0}$ bar, and $\gamma=c_{p} / c_{v}=1.2$ (at high temperatures).

Answer. Since the explosion takes place very rapidly, the expansion is adiabatic with $P V^{\gamma}=C$, a constant. The energy $Y$ of the weapon does work on the air in the cavity,

$$
\begin{aligned}
& Y=\int-P d V=\int-C V^{-\gamma} d V=C V^{1-\gamma} /(\gamma-1), \\
& Y=P_{0} V /(\gamma-1)=P_{0}\left(4 \pi a_{c}^{3} / 3\right) /(\gamma-1) .
\end{aligned}
$$

Using the values $Y=1 \mathrm{kt}, \gamma=1.2$, and $P_{0}=440 \mathrm{bar}$, we obtain a value for the minimum elastic radius, $a_{c}=16 \mathrm{~m}$, which agrees with the value calculated ${ }^{9,10}$ for a deep cavity several $\mathrm{km}$ below the surface. For $Y=1 \mathrm{Mt}, a_{c}=160 \mathrm{~m}$, and $V$ would be 1000 times larger.

\section{High frequencies}

The frequency spectrum of an explosion $(U)$ is obtained by Fourier transforming the elastic displacement produced by a step pressure at a distance $(r)$ from the explosion in a uniform medium, ${ }^{9,10}$

$$
U(\omega)=\left(a^{3} P_{0} v\right)\left[(c r)\left(v^{2}-0.75 a^{2}+i v a \omega\right)\right],
$$

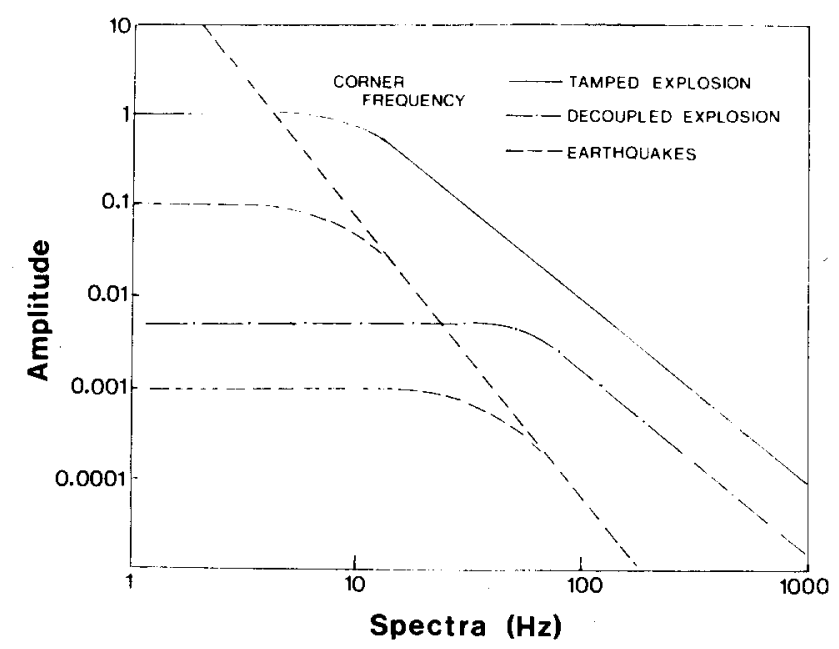

Fig. 2. High-frequency seismic detection. Relative seismic amplitudes as a function of frequency of earthquakes, and nuclear weapons explosions ( 1 kt decoupled, and tamped). The detection of the seismic signals from explosions are enhanced with respect to earthquakes at higher frequencies. Figure courtesy of J. Evernden. where the compressional wave velocity $v=4 \mathrm{~km} / \mathrm{s}$ and $c$ is a constant. Comment on the high- and low-frequency limits of $U(\omega)$.

Answer. Since the low-frequency limit of Eq. (1),

$$
U(\omega<v / a)=P_{0} a^{3} / c u r,
$$

is not a function of $\omega$, the seismic frequency is constant until it drops (Fig. 2) at the "corner frequency." Since the energy $Y$ of the weapon is proportional to $P_{0} a^{3}, U(\omega)$ is proportional to $Y$ and does not depend on $a$ as long as $a>a_{c}$. This is the basis of Latter's statement ${ }^{10}$ that the size of the cavity does not affect the distant signal as long as the radius of the cavity is greater than the decoupling radius $a_{c}$.

The high-frequency limit of $U(\omega)$ is proportional to $w^{-2}$. Since the corner frequency of an explosion is greater than the corner frequency of an earthquake with the same spectral level at low frequencies, the signal from an explosion is enhanced with respect to earthquakes and microseismic noise at the higher frequencies. Thus the spectrum above about $30 \mathrm{~Hz}$ can help distinguish nuclear tests from earthquakes and seismic noise.

\section{OPTICAL RECONNAISSANCE}

The optical systems for reconnaissance have improved ${ }^{11,12}$ considerably in a variety of ways: (1) Commerical aerial survey films are now available with a high contrast capability of about 1000 line pairs per $\mathrm{mm}$, or about 1 $\mu$ between lines. (2) Chromatic aberration has been reduced with the aid of computer-aided design and machining of lenses. (3) The blurring of a photograph by the forward motion of a satellite has been removed by slightly moving the film relative to the lens with precise timing mechanisms and servo-control systems. (4) Similar objects may reflect and emit differently in the visible and infrared wavelength regions. These differences can be studied with multispectral cameras using multiple lens systems which take multiple (at least nine) photographs at different wavelengths of the same object. (5) By using two views of the same terrain taken at different angles, one can obtain stereoscopic images which resolve the heights of objects. Such a cartographic camera in the space shuttle can cover an area of $25000 \mathrm{~km}^{2}$ in stero with a resolution of about 10 $m$ by using only a single pair of pictures. (6) Fourier transform infrared spectroscopy can rapidly and accurately determine trace impurities in the air. In this paper we will discuss progress in four additional reconnaissance areas, namely for charge-coupled devices (CCD), adaptive optics, digital image processing, and infrared.

\section{A. Film versus CCDs}

Compare the approximate resolutions available with a $\mathrm{CCD}^{13}$ with a pixel size of $10 \mu$ and films of 100 and 1000 lines $/ \mathrm{mm}$. Assume a camera focal length $(f)$ of 6 , a lens diameter $(D)$ of $1 \mathrm{~m}$, and a camera altitude of $120 \mathrm{~km}$. Some have said that it is possible to read the masthead of the newspaper Pravda from a satellite; is this possible?

Answer. Since the object distance $(o=120 \mathrm{~km})$ is much greater than the focal length ( $f=6 \mathrm{~m}$ ), the image distance is $i=f=6 \mathrm{~m}$. The resolution of the image is about $10 \mu$ $\left(h_{i}\right)$ for the film of 100 lines $/ \mathrm{mm}\left(1\right.$ line $\left./ 10^{-5} \mathrm{~m}\right)$. This corresponds to an object resolution of

$h_{0}=h_{i}(o / i)=\left(10^{-5} \mathrm{~m}\right)\left(12 \times 10^{4} \mathrm{~m} / 6 \mathrm{~m}\right)=20 \mathrm{~cm}$. 
The angular spread from diffraction effects since the diffraction limit for a $1-\mathrm{m}$ lens is about

$\theta_{d}=1.2 \lambda / D=(1.2)\left(5 \times 10^{-7} \mathrm{~m}\right) /(1 \mathrm{~m})=0.6 \mu \mathrm{rad}$.

The value of $1.2 \lambda / D$ is a reasonable compromise between the full width between the diffraction minima $(2.4 \lambda / D)$ and the full width at half-maximum intensity $(0.8 \lambda / D)$. The diffraction broadening can be neglected here because $\theta_{d}$, the diffraction limit, is much less than the angular uncertainty from the $10-\mu$ film,

$$
\theta_{f}=H_{i} / i=10^{-5} / 6=1.7 \mu \mathrm{rad} \text {. }
$$

The 1000 -lines $/ \mathrm{mm}$ film $\left(h_{i}=1 \mu\right)$ would introduce an angular spread of $0.17 \mu \mathrm{rad}$, but this film and camera combination does not give the theoretical resolution of $h_{0}=2$ $\mathrm{cm}$ because the diffraction spread of $\theta_{d}=0.6 \mu \mathrm{rad}$ exceeds the film spread of $\theta_{f}=0.17 \mu \mathrm{rad}$. (Real systems may achieve resolutions about midway between these two extreme cases, or, perhaps, about $10 \mathrm{~cm}$.) To improve the resolution to the limit caused by atmospheric turbulence (Sec. III B), it is desirable to go to larger lenses, as for example the 2.4-m mirror of the space telescope.

CCDs are in many ways much more attractive than film for photo-reconnaissance because they can be used in realtime with computers, they can be reused (the amount of available film often determines the useable life of a photographic satellite), they are sensitive to a broader range of frequencies, they have a much greater dynamic range between black and white, their response is extremely linear compared to the nonlinearity of film, and they are about 70 times more efficient in using light than film. CCDs will be used in the space telescope $(1600 \times 1600$ pixels, about $10 \mu$ in size) to obtain resolutions of about 0.3 to $0.5 \mu \mathrm{rad}$. However, the larger pixel size of CCDs is exacerbated by an additional factor of about 2 since it takes about 2 to 2.5 pixels to obtain the same ground information as one line pair from film. Thus it will be necessary to use CCDs with longer focal length (or folded optics) cameras. [Since the better resolutions $(2 \mathrm{~cm})$ from the better films ( 1000 lines/ $\mathrm{mm}$ ) are unobtainable because of diffraction limitations, the CCDs would not have to compete with that situation by having a focal lengths of ( 10 for pixel) ( 2 for $C C D)=20$ times longer.] Ultimately, the criteria for the use of CCDs rests on (1) what resolution is necessary to do the job and (2) data handling ability. If the resolution of a reconnaissance satellite was $10 \mathrm{~cm}$, it should not be possible to read the Pravda masthead, but it should be possible to distinguish a VW Beetle from a Chevrolet Vega. It should be possible to obtain sufficient of resolutions with CCDs by increasing the focal length of $6 \mathrm{~m}$ by a factor of about 5 . Because of their many advantages, CCDs are becoming very important for verification even if long focal length systems have to be built, or if some resolution has to be sacrificed. By taking occasional CCD pictures of large areas and subtracting them in the computer from previous pictures taken in the same season, changes in the scenery can be observed.

\section{B. Looking downward versus upward}

Stars twinkle at night because of the variations in refraction in the air cells caused by turbulence in the atmosphere. The problem of looking downward from a satellite is considerably smaller than looking upward (the "seeing") from a telescope, which is about $5 \mu \mathrm{rad}(1 \mathrm{arcsec})$. The "seeing" looking downward should be less since the satellites are more than 10 times further from the turbulence. How might the asymetries of this phenomena be illuminated using (1) the simple lens formula, (2) an optical bench, and (3) a shower curtain.

Answer. (1) Using a $\frac{1}{2}$-m converging lens, establish an image of an object with some fine detail, such as a wire mesh, that is about $5 \mathrm{~m}$ from the lens. Place a bunsen burner first near the object, and then near the lens. Note that the turbulence from the burner does not noticably distort the image when it is close to the object, but that it does distort the image when it is placed near the lens. (2) Apply the thin lens equation to a two-lens system in which the first lens has a long focal length, implying turbulence in the Earth's atmosphere, and the second lens is the camera lens. By slightly shifting the location (or its focal length) of the first lens, the position of the final image is slightly shifted. For the reconnaissance case, when the first lens ( the atmosphere) is close to the object (the missile silo) and far from the second lens (the reconnaissance camera), the effect of shifting the lens is very small (second order ${ }^{14}$ ). For the astronomical case, when the first lens (the atmosphere) is close to the second lens (the telescope) and far from the object (the stars), the correction for the final image location is larger (first order). (3) The next time you are taking a shower with a translucent shower curtain, observe that you are unable to see objects clearly through the curtain, but if you look over the curtain into a distant mirror, you will be able to see quite clearly your hand near the curtain.

\section{Adaptive optics}

For the case of large diameter cameras, atmospheric turbulence is not as significant a problem for reconnaissance satellites as it is for taking pictures from the earth of astronomical objects and satellites in orbit. The technique ${ }^{15}$ of adaptive optics (AO) has been used to improve the resolution of Earth-based telescopes to nearly the diffraction limit. Adaptive optics uses wave front sensors and wave front processors to determine distortions (the slope) in the wave front of the incoming light; this information is used to deform a mirror to correct for the misalignment of the wave front. (1) If the turbulent air cells in the troposphere at an altitute of $10 \mathrm{~km}$ have a size of about 10 to $20 \mathrm{~cm}$, would adaptive optics be useful for observing objects in orbit from the Earth? (2) What is the limit to the angular resolution for cameras on the earth and in orbit caused by turbulence? (3) How deformable should the AO mirror be? (4) How good must the tracking for $\mathrm{AO}$ be to observe objects in orbit from the Earth?

Answer. (1) Turbulence in the tropopause $(10 \mathrm{~km})$ causes angular blurring at the Earth-based telescope of about

$$
\theta_{t}=0.1 \mathrm{~m} / 10 \mathrm{~km}=5-10 \mu \mathrm{rad} .
$$

This is considerably larger than the diffraction limit of 0.6 $\mu$ rad for a $1-\mathrm{m}$ mirror (Sec. III A), hence AO can be useful for monitoring objects in orbit. (2) Since reconnaissance satellites are more than ten times further away from the troposphere, $\theta_{t}$ is about $0.5-1 \mu \mathrm{rad}$; therefore, $\mathrm{AO}$ is presently of marginal use for reconnaissance satellites. Only for mirrors larger than about $1 \mathrm{~m}$ and for the best films would AO become attractive enough to warrant the effort for photography in low-Earth orbit. (3) Since the wavelength of visible light is about $0.5 \mu$, a deformable mirror must pro- 
vide about $1 \mu$ of compensation with a precision of about 10 $\mathrm{nm}(1 \%)$. (4) The computer systems must be able to respond within about $1 \mathrm{~ms}$ since the period of the atmospheric disturbances is about $20 \mathrm{~ms}$. Since a satellite moves at about $8000 \mathrm{~m} / \mathrm{s}$, it would move during that time $(1 \mathrm{~ms}$ ) $(8000 \mathrm{~m} / \mathrm{s})=8 \mathrm{~m}$. It would be necessary to have extremely accurate tracking of about $1 \%(8 \mathrm{~cm} / \mathrm{ms})$ of this value for the AO telescopes to retain a resolution of $10 \mathrm{~cm}$.

\section{Digital image processing}

The advent of electro-optical devices such as CCDs and very large scale integrated circuits (VLSIC) enhances the ability to monitor military activities. Digital image processing (DIP) techniques ${ }^{16}$ can restore and enhance photographs that have been blurred by a variety of causes. DIP can be used to subtract one picture from another picture taken at a later time to extract changes in the scenery during the elapsed time. It can enhance edges and lines in an image, remove noise from the picture, enhance contrasts such as partially removing clouds, and search for various patterns such as circular missile silos or movable SS-20 missiles launchers. These techniques have been applied to a wide variety of fields such as photography, radar, sonar, and medicine. The formation of an image $(g)$ involves an image spreading function

$$
\begin{aligned}
g(x, y)= & \int_{-\infty}^{\infty} \int_{-\infty}^{\infty} h_{c}\left(x-x_{1}, y-y_{1}\right) \\
& \times s\left(x_{1}, y_{1}\right) d x_{1} d y_{1}+n(x, y),
\end{aligned}
$$

where $h_{c}$ is the composite point-spread function (caused by object or image motion, distortions by the detector, and aberrations by the optical system ), $s$ is the direct and indirect flux at the sensor, and $n$ is the noise. The information that we would like to extract from the image to obtain the pure signal is compounded because the point-spread function is multiplicative and the noise is additive. It is possible to improve the extraction process by analyzing the image in the frequency domain through Fourier transforms.

\section{The convolution theorem}

If we ignore the noise term in Eq. (2), its Fourier transform to the frequency domain is

$$
G(u, v)=H(u, v) S(u, v),
$$

where $G, H$, and $S$ are the two-dimensional Fourier transforms of the image $(g)$, the point-spread function $\left(h_{c}\right)$, and the signal $(s)$ at the detector. The beauty of the convolution theorem is that the equation in the frequency domain is less complex since it is multiplicative without the integration operations. As a simple application, consider a one-dimensional photograph of very narrow spots of light from two lasers which can be represented by delta functions. (1) If one observes an image

$$
g(x)=N\left(A e^{-x^{2} / 2 \sigma^{2}}+B e^{-(x-D)^{2} / 2 \sigma^{2}}\right),
$$

where are the lasers, what are their intensities, and what is $h_{c}$ ? (2) How could $h_{c}$ be determined for an optical system in orbit as it takes pictures of objects on the ground?

Answer. If we assume a Gaussian point-spread function

$$
h_{c}=N e^{-\left(x-x_{1}\right)^{2} / 2 \sigma^{2}}
$$

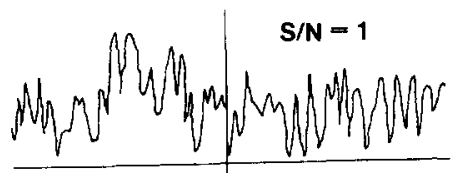

(b)

G(k)

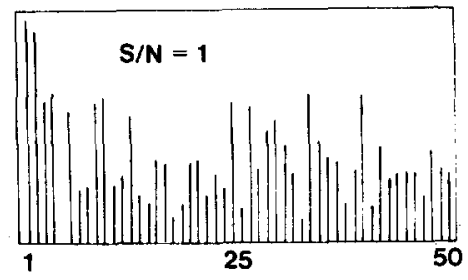

(c)

$s(x)$

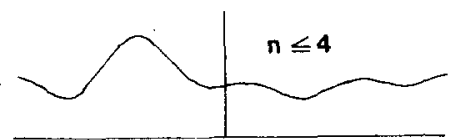

Fig. 3. Fourier transform. (a): A one-dimensional silo 20 pixels wide is placed in a field 100 pixels wide. The photographic data, $g(x)$, indicates that the silo is severely hidden by the large signal to noise ratio $(S / N=1)$. (b) : The spatial data is Fourier transformed to obtain its frequency spectrum: $F[g(x)]=G(k)$. (c): The noise is removed from the photo by Fourier transforming only the lowest four frequency components back to position space to make the "silo" more apparent: $F[G(k)]=s(x)$ for $n$ only from $1-4$.

with $N=\left(2 \pi \sigma^{2}\right)^{-1 / 2}$, one obtains

$$
g(x)=N \int_{-\infty}^{\infty} e^{-\left(x-x_{1}\right)^{2} / 2 \sigma^{2}}[A \delta(0)+B \delta(D)] d x_{1} .
$$

Since this integral gives Eq. (4), our choice for $h_{c}$ was correct. The intensities of the lasers are $A$ and $B$, and they are located at 0 and $D$. (2) For the case of a real satellite system, the function $h_{c}$ can be obtained by pointing a laser from the Earth to the satellite. The data $(g)$ and the known signal $(s)$ are Fourier transformed to give the Fourier transform of $h_{c}$ [Eq. (3), $H=G / S$ ]. Fourier transforming $H$ to real space gives $h_{c}$; this function includes not only the point-spread function of the system, but also the turbulence effects which vary with time.

\section{The addition theorem}

A picture of a silo can be partially hidden by a lack of contrast and photographic noise. In the case when the optical resolution is small compared to the size of the silo, one can ignore the point-spread function $\left(h_{c}\right)$; Eq. (2) becomes

$$
g(x)=s(x)+n(x)
$$

in one dimension. Since the diameter of the silo is much larger than the average wavelength of the noise, it will be possible to remove much of the noise in the frequency domain. The Fourier transform addition theorem is

$$
\begin{aligned}
F[g(x)] & =G(k)=F[s(x)+n(x)] \\
& =S(k)+N(k),
\end{aligned}
$$

where $F$ is the Fourier transform. How can one remove the noise from a one-dimensional picture,

$$
g(x)=N N \text { Rnd (1) }+s \text {, }
$$

where $N N$ is the peak noise, Rnd (1) is a random number 
from 0 to 1 , and the silo $(s)$ is placed in only part of the domain?

Answer. See Fig. 3 for the case of a silo 20 pixels wide located in a field 100 pixels wide with an average signal to noise ratio of $S / N=1$.

\section{INFRARED RECONNAISSANCE}

Infrared technologies have developed ${ }^{17}$ to the point where scanning devices routinely detect temperature differences of about $0.1 \mathrm{~K}$. Infrared pictures from satellites can be useful for a variety of verification tasks: counting "warm" missile silos to determine compliance with SALT, determing activity at nuclear production reactors and at enrichment and reprocessing facilities to monitor a freeze on the production of fissile isotopes ( $\mathrm{Pu}, \mathrm{U}-235$ ), to observe tests of space weapons (ASAT and BMD), and so forth. The infrared reconnaissance satellites detect a different kind of data (heat), but they are not as capable as those operating in the visible region at this time. The ir satellites use smaller arrays of up to 20000 detectors as compared to optical CCDs with arrays of 3 million detectors.

\section{A. Resolution}

There are two windows in the atmosphere which transmit ir, between 3 and $5 \mu$ and between 8 and $14 \mu$. For space based photography the 8 to $14-\mu$ window is often preferable because most of the Earth is at $300 \mathrm{~K}(10 \mu)$, and the 8 to $14-\mu$ window is further removed from reflected sunlight at $0.5 \mu$. (1) What is the maximum resolution in the 8 to $14-\mu$ region from a satellite at an altitude $(o)$ of $120 \mathrm{~km}$, using a mirror diameter $(D)$ of $1 \mathrm{~m}$ and folded, long-focal-length optics? (2) How would one have to modify Si to detect ir radition?

Answer. (1) Since the longer wavelengths for ir radiation increase the diffraction limit of the angular resolution, we will neglect the geometrical spreading from the size of the detector. For case of $14-\mu$ ir radiation with a $1-\mathrm{m}$ mirror, we obtain

$\theta_{d}=1.2 \lambda / D=(1.2)\left(14 \times 10^{-6} \mathrm{~m}\right) /(1 \mathrm{~m})=20 \mu \mathrm{rad}$,

which gives a mimimum spatial resolution of

$$
h_{0}=o \theta_{d}=(120 \mathrm{~km})(20 \mu \mathrm{rad})=2-3 \mathrm{~m} \text {. }
$$

This result is consistent with the resolutions obtained in recent astronomical ir measurements: The IRAS satellite observed a ring of solid material at an angle of $50 \mu \mathrm{rad}$ from the star Vegas, Mt. Polomar has obtained $4 \mu \mathrm{rad}$ in the ir, and the 4-m Kitt Peak telescope observed a "planet" $5 \mu \mathrm{rad}$ from the star VB-8. Infrared photography does not resolve objects as well as photography using visible light, but it is sufficient to detect silos and vehicles. (2) When an impurity such as $\mathrm{Ga}$ is incorporated into $\mathrm{Si}$, it has energy levels at about $0.1 \mathrm{eV}$ above the valence band which can be excited by the $10-\mu$ radiation since $E=1.24$. Semiconducting compounds with narrow bandgaps also are used to detect ir light.

\section{B. ir temperature sensitivity}

What accuracy is required to measure temperatures of objects near room temperature differences to within $\Delta T=0.01 \mathrm{~K}$ ?

Answer. Since the maximum of the blackbody curve (10 $\mu$ for $300 \mathrm{~K}$ ) is approximately in the center of the 8 - to $14-\mu$ window, the integral response of the detector is approximately proportional to $T^{4}$ for small changes in temperature $(\Delta T)$. (This can be shown with numerical integrations of the Planck distribution function.) The difference in accumulated charge or voltage is approximately proportional to

$$
\Delta\left(\sigma T^{4}\right)=\left(\sigma T^{4}\right)(4 \Delta T / T)
$$

when $\Delta T \ll T$. For $\Delta T=0.01 \mathrm{~K}$ and $T=300 \mathrm{~K}$, the required accuracy is about (4) $(0.01 \mathrm{~K}) / 300 \mathrm{~K}=0.01 \%$, i.e., the charge accumulated in the ir detector would have to be measured with an accuracy of one part in 10000 . The ir radiation emitted by the atmosphere gives a background radiation noise which tends to degrade the accuracy of the temperature measurement from a satellite.

\section{Detection of cool objects at a distance}

The quantum efficiency of a Si:Ga detector in the ir region is about 0.5. Determine if an ir detector can detect a cool $\left(273 \mathrm{~K}\right.$ ) object with a total area of $1 \mathrm{~m}^{2}$ (a satellite or a reentry vehicle) at a distance of $1000 \mathrm{~km}$. (1) What rate of charge collection could be obtained by a detector that uses a 1-m diameter mirror to enhance its effective area? About $35 \%$ of the blackbody radiation from an ice cube $(273 \mathrm{~K})$ falls within the 8- to $14-\mu$ window. Since the object is in space, the background radiation can be ignored. (2) What signal to noise $(\mathrm{S} / \mathrm{N}$ ) ratio (from the thermal noise in the detector) might be expected if the detectivity $(D)$ is about $5 \times 10^{12}$ where $D=(\mathrm{S} / \mathrm{N}) / P$, where $P$ is the ir power on the detector from the cool object in the 8- to 14- $\mu$ window. This value of $D$ was multiplied by a factor of 100 to account for detector size, amplifier bandwidth, and field of view.

Answer. (1) The object radiates (between 8 and $14 \mu$, in mks units)

$$
\begin{aligned}
P_{\text {ir }} & =(0.35)\left(\sigma T^{4} A\right) \\
& =(0.35)\left(5.7 \times 10^{-8}\right)\left(273^{4}\right)(1)=110 \mathrm{~W} .
\end{aligned}
$$

At a distance of $1000 \mathrm{~km}(R)$ the 1-m-diam $(D)$ mirror collects

$$
P=\left(P_{\text {ir }}\right)\left(D^{2} / 16 R^{2}\right)=7 \times 10^{-12} \mathrm{~W} .
$$

This corresponds to a photon (average photons of $11 \mu$, $0.11 \mathrm{eV}$ ) flux at the detector of $4 \times 10^{8}$ per second. Since the quantum efficiency is 0.5 , about $2 \times 10^{8}$ electrons/s could be collected by the detector. The signal to noise ratio would be about

$$
\mathrm{S} / \mathrm{N}=D P=\left(5 \times 10^{12}\right)\left(7 \times 10^{-12}\right)=35 \text {. }
$$

Since both the $\mathrm{S} / \mathrm{N}$ ratio and the charge collection rate are large, the detection time can be quite short.

\section{RADAR}

\section{A. Synthetic aperture radar (SAR)}

SAR systems ${ }^{18}$ mounted in satellites are presently used to obtain maps of features on the Earth and the planets as well as to monitor military activities. Because the wavelength of radar waves is longer than optical waves, the resolution from SAR is not as good as that obtained with optical systems. However, radar has the great attribute that it can be used during the night and when the Earth is covered with clouds, rain, or foliage. To obtain high resolution radar images of the Earth from a satellite would require an antenna several miles long. To circumvent this problem, the motion of the satellite or aircraft carrying the SAR 
system is used to increase the effective size of the antenna. A resolution of about $25 \mathrm{~m}$ has been obtained with the SEASAT satellite at an orbit height of $800 \mathrm{~km}$. Considerably better resolutions of 1-3 $\mathrm{m}$ are possible at a higher radar frequency of $10 \mathrm{GHz}$, and at lower altitudes. For example, consider the case of a radar operating at $6 \mathrm{GHz}$ ( $\lambda=0.05 \mathrm{~m}$ ) at a slant range of $R=200 \mathrm{~km}$. (1) What is the effective size of a SAR antenna needed to obtain a resolution $(\Delta R)$ of $5 \mathrm{~m}$ ? (2) What is the processing time to obtain this resolution?

Answer. (1) the angular resolution is approximately

$$
\text { (2) } \theta_{d}=\Delta R / R=25 \mu \mathrm{rad}=\lambda / 2 D \text {. }
$$

(The synthesized antenna produces an effective beam width from an antenna which is twice the synthetic antenna length.) This give a synthetic aperture of $D=1000$ m. (2) Since the orbital velocity is about $8 \mathrm{~km} / \mathrm{s}$, the processing time for the SAR system must be about $(1 \mathrm{~km} / 8$ $\mathrm{km} / \mathrm{s})=0.1 \mathrm{~s}$. Longer processing times would improve resolution in the tracking direction parallel to the velocity of the satellite, but it can never be better than $\frac{1}{2}$ of the width of the actual physical antenna. As in the case of optical systems, the best achievable resolutions would be limited by atmospheric inhomogeneities and radar stability. Threedimensional surface maps can be obtained by either placing two antennas on a satellite or airplane, or by comparing data from two successive passes over the region of interest.

\section{B. Missile coefficient}

In order to monitor "new types" of missiles under SALT, it is necessary to be able to determine some quantitative aspects of individual reentry vehicles (RV) from ICBMs. The accuracy of an RV will be increased if the drag forces are small compared to its weight since the drag force disturbs the trajectory in unpredictable ways. The ratio of an RV's weight $(W)$ to its effective area $\left(C_{D} A\right.$, where $C_{D}$ is the drag coefficient) is called its missile coefficient, $\beta=W / C_{D} A$, usually given in English units. How can radar be used to measure $\beta$, and thus monitor the accuracy of an RV?

Answer. By measuring the Doppler shift $(\Delta f)$ of the reflected radar signal, the velocity can be determined from

$$
\Delta f=2 v f / c \text {, }
$$

where $f$ is the radar frequency and $v$ is the velocity of the $\mathrm{RV}$. By measuring the change of $\Delta f$ with time, one can determine the deceleration of the $R V$ in the atmosphere.
The deceleration gives the ratio of the weight to the drag force $\left(a=F_{D} / m\right)$ to obtain $\beta$. The value of $\beta$ for an accurate $R V$ of $800 \mathrm{lb}$ with an area of $3 \mathrm{ft}$ and a drag coefficient $C_{D}$ of 0.15 is about

$\beta=W / C_{D} A=(800 \mathrm{lb}) /(0.15)\left(3 \mathrm{ft}^{2}\right)=1800 \mathrm{lb} / \mathrm{ft}^{2}$.

\section{ACKNOWLEDGMENT}

I would like to thank Dietrich Schroeer for a critical reading of the manuscript.

${ }^{1}$ W. Potter, Ed., Verification and SALT: The Challenge of Strategic Deception (Westview, Boulder, 1980); R. Scribner, W. Metz, and T. Ralston, Verification and Arms Control: A Primer on the Technology and Process of Verification (Birkhauser, Boston, 1985); F. Hussain, The Impact of Weapons Test Restrictions (Int. Inst. Strategic Studies, London, 1981); D. Hafemeister, Bull. At. Sci. 41, 35 (1985); W. Potter, Ed., Verification and Arms Control (Lexington, Lexington, MA, 1985); D. Hafemeister, J. Romm, and K. Tsipis, Sci. Am. 252, 38 (1985).

${ }^{2}$ D. Hafemeister, Am. J. Phys. 41, 1191 (1973); 42, 625 (1974); 44, 86 (1976); 47, 671 (1979); 48, 112 (1980); 50, 29 (1982); 50, 713 (1982); 51, 215 (1983).

${ }^{3}$ K. Tsipis, D. Hafemeister, and P. Janeway, Eds., Arms Control Verification: The Technologies That Make It Possible (Pergamon, Elmsford, NY, 1986).

${ }^{4}$ S. Glasstone and P. Dolan, The Effects of Nuclear Weapons (Dept. Defense and Energy, Washington, DC, 1977).

${ }^{5}$ H. Argo, Ref. 3.

${ }^{6}$ H., Brode, Ann. Rev. Nucl. Sci. 18, 153 (1968).

${ }^{7}$ P. Stokes, Ref. 3.

${ }^{8}$ L. Sykes, J. Evernden, and I.Cifuentes, AIP 104, 85 (1983); W. Hannon, Science 227, 251 ( 1985 ).

${ }^{9} \mathrm{~J}$. Evernden and C. Archambeau, Ref. 3.

${ }^{10}$ R. Latter, R. Herbst, and K. Watson, Ann. Rev. Nucl. Sci. 11, 371 (1961); O. Dahlman and H. Isrealson, Monitoring Underground Nuclear Explosions (Elsevier, New York, 1977).

${ }^{11}$ J. Richelson, J. Strat. Stud. 7, 121 (1984).

${ }^{12}$ R. Ondrejka, Ref. 3.

${ }^{13}$ M. Blouke and J. Janesick, Ref. 3; K. Kristian and M. Blouke, Sci. Am. 247, 67 (1982); J. Bahcall and L. Spitzer, Sci. Am. 247, 40 (1982).

${ }^{14} \mathrm{~T}$. Foster ane D. Hafemeister (to be published).

${ }^{15} \mathrm{~J}$. Vyce and J. Hardy, Ref. 3.

${ }^{16}$ B. Hunt, Ref. 3; H. Andrews and B. Hunt, Digital Image Restoration (Prentice-Hall, Englewood Cliffs, NJ, 1977).

${ }^{17}$ J. Fraser, Ref. 3; A. Kahle and A. Goetz, Science 222, 24 (1983); H. Levinson, Phys. Today 30, 23 (Nov., 1977).

${ }^{18} \mathrm{~J}$. Constant, Introduction to Defense Radar Systems Engineering (Macmillian, London, 1972); E. Brookner, Radar Technology (Artech, Dedham, MA, 1977); E. Brookner, Ref. 3. 